\title{
NON-REGULARITY IN HÖLDER AND SOBOLEV SPACES OF SOLUTIONS TO THE SEMILINEAR HEAT AND SCHRÖDINGER EQUATIONS
}

\author{
THIERRY CAZENAVE ${ }^{1}$, FLÁVIO DICKSTEIN $^{2}$, AND FRED B. WEISSLER $^{3}$
}

\begin{abstract}
In this paper we study the Cauchy problem for the semilinear heat and Schrödinger equations, with the nonlinear term $f(u)=\lambda|u|^{\alpha} u$. We show that low regularity of $f$ (i.e., $\alpha>0$ but small) limits the regularity of any possible solution for a certain class of smooth initial data. We employ two different methods, which yield two different types of results. On the one hand, we consider the semilinear equation as a perturbation of the ODE $w_{t}=$ $f(w)$. This yields in particular an optimal regularity result for the semilinear heat equation in Hölder spaces. In addition, this approach yields ill-posedness results for NLS in certain $H^{s}$ spaces, which depend on the smallness of $\alpha$ rather than the scaling properties of the equation. Our second method is to consider the semilinear equation as a perturbation of the linear equation via Duhamel's formula. This yields in particular that if $\alpha$ is sufficiently small and $N$ sufficiently large, then the nonlinear heat equation is ill-posed in $H^{s}\left(\mathbb{R}^{N}\right)$ for all $s \geq 0$.
\end{abstract}

\section{INTRODUCTION}

This paper is concerned with regularity of solutions of two well known and well studied semilinear evolution equations, the semilinear heat equation

$$
\left\{\begin{array}{l}
u_{t}=\Delta u+\lambda|u|^{\alpha} u \\
u(0, \cdot)=u_{0}(\cdot)
\end{array}\right.
$$

and the semilinear Schrödinger equation

$$
\left\{\begin{array}{l}
i u_{t}=\Delta u+\lambda|u|^{\alpha} u \\
u(0, \cdot)=u_{0}(\cdot)
\end{array}\right.
$$

in $\mathbb{R}^{N}$, where $\alpha>0$ and $\lambda \in \mathbb{C}, \lambda \neq 0$. More precisely, we allow the initial value $u_{0}$ to be infinitely smooth, and we study the loss of regularity due to the nonlinear term. Therefore, we are particularly interested in small values of $\alpha>0$. Let $f(u)=|u|^{\alpha} u$ with $0<\alpha<1$. As a point function $f$ is $C^{1}$ but not $C^{2}$. Formally, this might be considered an obstacle to the regularity of solutions of (1.1) and (1.2). Indeed, in order to prove the regularity of the solutions of (1.1) and (1.2) (for instance by a fixed-point argument), one uses the regularity of the nonlinear

2010 Mathematics Subject Classification. Primary 35B65; secondary 35A01, 35B33, 35K91, 35Q55, 35Q56.

Key words and phrases. Non-regularity of solutions, ill-posedness, semilinear heat and Schrödinger equations.

Research supported by the "Brazilian-French Network in Mathematics".

Flávio Dickstein was partially supported by CNPq (Brasil), and by the Fondation Sciences Mathématiques de Paris. 
term. However, the relationship between the regularity of $f$ and the regularity of the solution is not a simple one. Indeed, suppose $u$ is $C^{2}\left(\mathbb{R}^{N}, \mathbb{C}\right), u\left(x_{0}\right)=0$ and $\nabla u\left(x_{0}\right) \neq 0$ for some $x_{0} \in \mathbb{R}^{N}$, then $f(u) \notin C^{2}\left(\mathbb{R}^{N}, \mathbb{C}\right)$. On the other hand, for any reasonable initial value, for example in $C_{0}\left(\mathbb{R}^{N}\right)$, the corresponding solution of (1.1) will in fact be $C^{2}$ in space for $t>0$ by standard parabolic regularity. Thus the non-regularity of $f(u)$ does not immediately imply the non-regularity of $u$.

The question of regularity is strongly related to the question of well-posedness. Recall that an evolution equation, such as (1.1) or (1.2), is locally well-posed in a Banach space $X$ if for every $u_{0} \in X$ there exist $T>0$ and a solution $u \in C([0, T], X)$ such that $u(0)=0$. In addition, the solution is required to be unique in some sense, not always in $C([0, T], X)$, and is also required to depend continuously, again in some appropriate sense, on the initial value $u_{0}$. The key point for our purposes is that if $X$ is a positive order Sobolev space, whose elements have a certain degree of regularity, the resulting solution maintains this regularity.

Specifically, if we wish to use a standard perturbation argument to prove that the Cauchy problem for either equations (1.1) or (1.2) is locally well-posed in $H^{s}\left(\mathbb{R}^{N}\right)$ for some given $s>0$ we are confronted with two different requirements on $\alpha$. On the one hand, we need that the nonlinear term be controlled by the linear flow. This translates (formally) as the condition

$$
\begin{cases}0<\alpha \leq \frac{4}{N-2 s}, & s<N / 2 \\ 0<\alpha<\infty, & s \geq N / 2\end{cases}
$$

On the other hand, in order to carry out the perturbation argument in $H^{s}$, the nonlinear term must be sufficiently smooth. When $\alpha$ is not an even integer, then $\alpha$ must be large enough so that $f(u)=|u|^{\alpha} u$ be sufficient regular. In the case of the simplest perturbation argument requiring an estimate of $|u|^{\alpha} u$ in $H^{s}\left(\mathbb{R}^{N}\right)$, this leads to the condition

$$
[s]<\alpha .
$$

See e.g. $[18,15]$ for the heat equation, $[11,12,8,13,17,10]$ for the Schrödinger equation.

Since the first condition (1.3) is related to scaling properties of the equation (see Section 3.1 in [19], and in particular the discussion p. 118), it can be considered as natural. In fact, in some cases it is known that if this condition is not satisfied, then the problem is not well-posed in $H^{s}\left(\mathbb{R}^{N}\right)$. See for instance $[15,9,5,6,2]$. On the other hand, (1.4) might appear as a purely technical condition which one should be able to remove by a more appropriate argument. Indeed, one can sometimes improve condition (1.4) by using the fact that one time derivative is like two space derivatives, but we are still left with the condition

$$
[s]<2 \alpha .
$$

See $[12,17,10]$.

The purpose of this paper, as opposed to the above cited papers, is to show that in certain cases "technical" restrictions such as (1.4) and (1.5) are not purely technical, but impose genuine limitations on the regularity of the solution. More precisely, we show that condition (1.4) is not always sufficient to imply local well-posedness of (1.1) and (1.2) in $H^{s}$. (See Remarks 1.3 and 1.6, and Theorem 1.10.) In fact, we prove under various circumstances that there exist initial values $u_{0} \in C_{\mathrm{c}}^{\infty}\left(\mathbb{R}^{N}\right)$ for 
which (1.1) or (1.2) cannot have a local solution with a certain degree of regularity. To our knowledge, there are no previous results of this type.

Our first result concerns the nonlinear heat equation (1.1), and is in fact optimal. We recall that the Cauchy problem (1.1) is locally well-posed in $C_{0}(\mathbb{R})$, i.e. for any $u_{0} \in C_{0}(\mathbb{R})$, there exist a maximal existence time $T_{\max }>0$ and a unique solution $u \in C\left(\left[0, T_{\max }\right), C_{0}(\mathbb{R})\right)$ of $(1.1)$. Let $0<\alpha<1$, let $u_{0}$ be smooth, and let $u \in C\left(\left[0, T_{\max }\right), C_{0}\left(\mathbb{R}^{N}\right)\right)$ be the resulting, maximal solution of (1.1). It is known that, given any $0<T<T_{\max }, \partial_{t} u, \nabla \partial_{t} u$, and all space-derivatives of $u$ of order up to 3 belong to $C\left([0, T] \times \mathbb{R}^{N}\right)$. Furthermore, the spatial derivatives of order 3 are $\alpha$-Hölder continuous, i.e.

$$
\sup _{\substack{0 \leq t \leq T \\|m|=3}}\left|\partial_{x}^{m} u(t)\right|_{\alpha}<\infty
$$

where $|w|_{\ell}$ is defined by

$$
|w|_{\ell} \stackrel{\text { def }}{=} \sup _{x \neq y} \frac{|u(x)-u(y)|}{|x-y|^{\ell}}
$$

for $\ell>0$ and $w \in C\left(\mathbb{R}^{N}\right)$. See Theorem A.1 in the Appendix for a precise statement. The theorem below shows that the $\alpha$-Hölder continuity cannot in general be improved in the sense that one cannot replace $\alpha$ by $\beta>\alpha$.

Theorem 1.1. Let $0<\alpha<1$ and $\lambda \in \mathbb{C} \backslash\{0\}$. There exists an initial value $u_{0} \in$ $C_{\mathrm{c}}^{\infty}\left(\mathbb{R}^{N}\right)$ such that the corresponding maximal solution $u \in C\left(\left[0, T_{\max }\right), C_{0}\left(\mathbb{R}^{N}\right)\right)$ of (1.1) is three times continuously differentiable with respect to the space variable and

$$
\int_{s}^{t}\left|\partial_{y} \Delta u(\sigma)\right|_{\beta}=+\infty
$$

for all $\alpha<\beta \leq 1$ and all $0 \leq s<t<T_{\max }$ with $t$ sufficiently small.

Theorem 1.1 has the immediate following corollary, by using Sobolev's embedding theorem (see (1.15)).

Corollary 1.2. Let $0<\alpha<1$. There exists an initial value $u_{0} \in C_{\mathrm{c}}^{\infty}\left(\mathbb{R}^{N}\right)$ such that the corresponding maximal solution $u \in C\left(\left[0, T_{\max }\right), C_{0}\left(\mathbb{R}^{N}\right)\right)$ of $(1.1)$ does not belong to $L^{1}\left((0, T), H^{s, p}\left(\mathbb{R}^{N}\right)\right)$ if $s>3+\frac{N}{p}+\alpha, 1<p<\infty$, and $0<T<T_{\max }$.

Remark 1.3. The initial value $u_{0} \in C_{\mathrm{c}}^{\infty}\left(\mathbb{R}^{N}\right)$ can be chosen arbitrarily small (in any space). See Remark 3.4. Corollary 1.2 therefore implies that $s \leq 3+\frac{N}{p}+\alpha$, $1<p<\infty$ is a necessary condition for (1.1) to be locally well posed in $H^{s, p}\left(\mathbb{R}^{N}\right)$ ), even in an arbitrarily small ball.

Remark 1.4. We should observe that formula (1.8) does not imply that $\left|\partial_{y} \Delta u(t)\right|_{\beta}$ is infinite for any given value of $0<t<T_{\max }$. On the other hand, it is stronger than saying that $\sup _{0 \leq t \leq T}\left|\partial_{y} \Delta u(t)\right|_{\beta}<\infty$. Similarly, Corollary 1.2 does not guarantee that $u(t) \notin H^{s}\left(\mathbb{R}^{N}\right)$ for any given value of $0<t<T_{\max }$.

Before stating our next result, we make some comments on the proof of Theorem 1.1. The key idea is to consider equation (1.1) as a perturbation of the ordinary differential equation

$$
w_{t}=\lambda|w|^{\alpha} w
$$

with the same initial condition $w(0, \cdot)=u_{0}(\cdot)$. As we shall see by a straightforward calculation (see Section 2), equation (1.9) produces a loss of spatial regularity. For 
example, in dimension $N=1$, if $u_{0}(x)=x$ in a neighborhood of 0 , then the resulting solution $w(t, x)$ of (1.9) will not be twice differentiable at $x=0$ for $t>0$. Moreover, for the perturbed equation

$$
w_{t}=\lambda|w|^{\alpha} w+h
$$

where $h$ is sufficiently smooth, the same loss of regularity occurs. (See Theorem 2.1.) Let now $u$ be a solution of the nonlinear heat equation (1.1), and set $h=\Delta u$. It follows that $u_{t}=\lambda|u|^{\alpha} u+h$. Thus we see that for appropriate $u_{0}$, if $h$ is sufficiently smooth, then $u(t)$ is not $C^{2}$ in space for small $t>0$. Since we know $u(t)$ is $C^{2}$ for $t>0$, this implies that $h=\Delta u$ is not too regular. Applying the precise regularity statement of Theorem 2.1 gives the conclusion of Theorem 1.1.

It turns out that the same arguments can be used to prove ill-posedness for the nonlinear Schrödinger equation (1.2). This yields the following analogue of Corollary 1.2.

Theorem 1.5. Let $0<\alpha<1, \lambda \in \mathbb{C} \backslash\{0\}$ and suppose $s>3+\frac{N}{2}+\alpha$. There exists $u_{0} \in C_{\mathrm{c}}^{\infty}\left(\mathbb{R}^{N}\right)$ such that there is no $T>0$ for which there exists a solution $u \in C\left([0, T], H^{s}\left(\mathbb{R}^{N}\right)\right)$ of $(1.2)$.

Remark 1.6. Theorem 1.5 implies that $s \leq 3+\frac{N}{2}+\alpha$ is a necessary condition for (1.2) to be locally well posed in $H^{s}\left(\mathbb{R}^{N}\right)$ ), even in an arbitrarily small ball. See Remark 3.4.

Theorem 1.5 turns out to be a specific case of an analogous result for the complex Ginzburg-Landau equation, see Theorem 3.2 below.

As pointed out in Remark 1.4, Theorem 1.1 and Corollary 1.2 do not guarantee the lack of spatial regularity of the solution $u$ of (1.1) at any fixed $t>0$. The following theorem gives an example of loss of spatial regularity for every $t>0$.

Theorem 1.7. Let $0<\alpha<2$ and $\lambda \in \mathbb{C} \backslash\{0\}$. There exists an initial value $u_{0} \in$ $C_{\mathrm{c}}^{\infty}\left(\mathbb{R}^{N}\right)$ such that the corresponding maximal solution $u \in C\left(\left[0, T_{\max }\right), C_{0}\left(\mathbb{R}^{N}\right)\right)$ of (1.1) satisfies $u(t) \notin H^{s, p}\left(\mathbb{R}^{N}\right)$ for all sufficiently small $0<t<T_{\max }$ if $1<p<$ $\infty$ and $s>5+\frac{1}{p}$.

Remark 1.8. As observed for previous results, the initial value $u_{0} \in C_{\mathrm{c}}^{\infty}\left(\mathbb{R}^{N}\right)$ in Theorem 1.7 can be chosen arbitrarily small (in any space). See Remark 4.3.

Unlike the proof of Theorem 1.2, the proof of Theorem 1.7 treats the nonlinear term $|u|^{\alpha} u$ as a perturbation of the linear heat equation, via the standard Duhamel formula. More precisely, for appropriate initial values we show that the integral term

$$
\mathcal{I}=\int_{0}^{t} e^{(t-s) \Delta}|u(s)|^{\alpha} u(s) d s
$$

can never be in $H^{s, p}\left(\mathbb{R}^{N}\right)$ if $s>5+\frac{1}{p}$. One key idea in the proof is to express $\left|u\left(t, x^{\prime}, y\right)\right|^{\alpha} u\left(t, x^{\prime}, y\right)=C\left(x^{\prime}\right) \gamma(t)|y|^{\alpha} y+\widetilde{w}\left(t, x^{\prime}, y\right)$ where $x^{\prime} \in \mathbb{R}^{N-1}$ and $y \in \mathbb{R}$, with $\left|\widetilde{w}\left(t, x^{\prime}, y\right)\right| \leq C|y|^{\alpha+2}$. This decomposition enables us to explicitly compute $\partial_{y}^{5} e^{\varepsilon \Delta} \mathcal{I}$ at $y=0$, which (if $\alpha<2$ ) goes to $\infty$ as $\varepsilon \downarrow 0$, uniformly for $x^{\prime}$ in a neighborhood of 0 . This shows that $\mathcal{I}$ cannot be $C^{5}$ with respect to $y$, and the result then follows from the one-dimensional Sobolev embedding theorem. We insist on this last point; since the proof is based on a one-dimensional argument, the condition on $s, p$ in the statement of Theorem 1.2 is independent of the space dimension $N$. 
On the other hand, in Corollary 1.2 the condition on $s, p$ does depend on the space dimension, since we deduce the result from Theorem 1.1 by the $N$-dimensional Sobolev embedding theorem. This is perhaps only a technical problem. Indeed, the proof of Theorem 1.1 is also based on a one-dimensional argument. However, the structure of that proof, via an argument by contradiction, does not seem to allow the application of the one-dimensional Sobolev embedding theorem.

For our last result, we introduce a very weak notion of local well-posedness for small data, which is weaker than the general notion described earlier in the introduction. Recall that (1.1) is locally well-posed in $C_{0}\left(\mathbb{R}^{N}\right)$, and $T_{\max }\left(u_{0}\right)$ is the maximal existence time of the solution corresponding to the initial value $u_{0}$.

Definition 1.9. Let $s \geq 0, \alpha>0$ and $\lambda \in \mathbb{C}$. We say that (1.1) is locally well posed for small data in $H^{s}\left(\mathbb{R}^{N}\right)$ if there exist $\delta, T>0$ such that if $u_{0} \in C_{\mathrm{c}}^{\infty}\left(\mathbb{R}^{N}\right)$ and $\left\|u_{0}\right\|_{H^{s}} \leq \delta$, then the corresponding solution $u \in C\left(\left[0, T_{\max }\right), C_{0}\left(\mathbb{R}^{N}\right)\right)$ of $(1.1)$ satisfies $T_{\max }\left(u_{0}\right) \geq T$ and $u(t) \in H^{s}\left(\mathbb{R}^{N}\right)$ for all $0 \leq t \leq T$.

Theorem 1.10. Let $0<\alpha<2$, and $\lambda \in \mathbb{R}$ with $\lambda>0$. If

$$
N>11+\frac{4}{\alpha}
$$

then for every $s \geq 0$, the Cauchy problem (1.1) is not locally well posed for small data in $H^{s}\left(\mathbb{R}^{N}\right)$.

The rest of the paper is organized as follows. We recall below the definitions of the various function spaces we use, and certain of their properties. In Section 2 we study regularity of solutions to the ordinary differential equation (1.9), and to the perturbed equation (1.10). In particular, we show (Theorem 2.1) that if $h$ is sufficiently smooth then (1.9) produces a singularity for a certain class of smooth data. In Section 3 we apply this result to prove Theorems 1.1 and 1.5, as well as a similar result for a complex Ginzburg-Landau equation (Theorem 3.2). In Section 4 we prove Theorems 1.7 and 1.10 .

One final remark about our results. Throughout this paper, we have considered small values of $\alpha$, either $0<\alpha<1$ or $0<\alpha<2$. It is likely that analogous results can be proved for larger values of $\alpha$.

Notation and and function spaces. Throughout this paper, we consider function spaces of complex-valued functions.

$L^{p}\left(\mathbb{R}^{N}\right)$, for $1 \leq p \leq \infty$, is the usual Lebesgue space, with norm $\|\cdot\|_{L^{p}}$. We denote by $C_{0}\left(\mathbb{R}^{N}\right)$ the space of continuous functions on $\mathbb{R}^{N}$ that vanish at infinity, equipped with the sup norm. $H^{s, p}\left(\mathbb{R}^{N}\right)$ and $H^{s}\left(\mathbb{R}^{N}\right)=H^{s, 2}\left(\mathbb{R}^{N}\right)$, for $s \geq 0$ and $1<p<\infty$ are the usual Sobolev spaces, and the corresponding norms are denoted by $\|\cdot\|_{H^{s}}$ and $\|\cdot\|_{H^{s, p}}$. In particular,

$$
\|u\|_{H^{s, p}}=\left\|\mathcal{F}^{-1}\left[\left(1+|\xi|^{2}\right)^{\frac{s}{2}} \widehat{u}\right]\right\|_{L^{p}}
$$

and $\|u\|_{H^{s, p}} \approx \sum_{|\ell| \leq s}\left\|\partial^{\alpha} u\right\|_{L^{p}}$ if $s$ is an integer. (See e.g. [4, Theorem 6.2.3].)

In the proof of Theorem 1.7, we use the property that if $u=u\left(x_{1}, x_{2}\right)$ with $x_{1} \in \mathbb{R}^{m}, x_{2} \in \mathbb{R}^{n}$, and if $1<p<\infty$ and $s \geq 0$, then

$$
\|u\|_{L_{x_{1}}^{p}\left(\mathbb{R}^{m}, H^{s, p}\left(\mathbb{R}_{x_{2}}^{n}\right)\right)} \leq C\|u\|_{H^{s, p}\left(\mathbb{R}^{m+n}\right)} .
$$

Inequality (1.14) with $C=1$ is immediate when $s$ is an integer. (The left-hand side has fewer terms than the right-hand side.) The general case follows by complex interpolation. Indeed, suppose $s$ is not an integer, fix two integers $0 \leq s_{0}<s<s_{1}$ 
and let $0<\theta<1$ be defined by $s=(1-\theta) s_{0}+\theta s_{1}$. It follows that $H^{s, p}\left(\mathbb{R}^{m+n}\right)=$ $\left(H^{s_{0}, p}\left(\mathbb{R}^{m+n}\right), H^{s_{1}, p}\left(\mathbb{R}^{m+n}\right)\right)_{[\theta]}$ and $H^{s, p}\left(\mathbb{R}^{n}\right)=\left(H^{s_{0}, p}\left(\mathbb{R}^{n}\right), H^{s_{1}, p}\left(\mathbb{R}^{n}\right)\right)_{[\theta]}$. (See $[4$, Theorem 6.4.5].) This last property implies that

$$
L^{p}\left(\mathbb{R}^{m}, H^{s, p}\left(\mathbb{R}^{n}\right)\right)=\left(L^{p}\left(\mathbb{R}^{m}, H^{s_{0}, p}\left(\mathbb{R}^{n}\right)\right), L^{p}\left(\mathbb{R}^{m}, H^{s_{1}, p}\left(\mathbb{R}^{n}\right)\right)\right)_{[\theta]} .
$$

(See [4, Theorem 5.1.2].) Estimate (1.14) now follows by complex interpolation between the estimates for $s=s_{0}$ and $s=s_{1}$.

We will use Sobolev's embedding into Hölder spaces. Recall definition (1.7). Given any $j \in \mathbb{N}$ and $0<\ell<1$, the Hölder space $C^{j, \ell}\left(\overline{\mathbb{R}^{N}}\right)$ is the space of functions $u$ whose derivatives of order $\leq j$ are all bounded and uniformly continuous, and such that $\left|\partial^{\gamma} u\right|_{\ell}<\infty$ for all multi-indices $\gamma$ with $|\gamma|=j . C^{j, \ell}\left(\overline{\mathbb{R}^{N}}\right)$ is a Banach space when equipped with the norm $\|u\|_{W^{j, \infty}}+\sum_{|\gamma|=j}\left|\partial^{\gamma} u\right|_{\ell}$. (See e.g. [1, Definition 1.29, p. 10].) Given $j \in \mathbb{N}, 0<\ell<1$ and $s \in(\ell+j, \ell+j+N)$, It follows that

$$
H^{s, p(s)}\left(\mathbb{R}^{N}\right) \hookrightarrow C^{j, \ell}\left(\overline{\mathbb{R}^{N}}\right)
$$

where $p(s)=\frac{N}{s-j-\ell} \in(1, \infty)$. Indeed, we may assume $j=0$, as the general case follows by iteration. Suppose first $s \geq 1$. Since $s-\frac{N}{p(s)}=\ell=1-\frac{N}{p(1)}$, it follows from [4, Theorem 6.5.1, p. 153] that $H^{s, p(s)}\left(\mathbb{R}^{N}\right) \hookrightarrow H^{1, p(1)}\left(\mathbb{R}^{N}\right)$. The result now follows from the embedding $H^{1, p(1)}\left(\mathbb{R}^{N}\right) \hookrightarrow C^{0, \ell}\left(\overline{\mathbb{R}^{N}}\right)$. (See $[1$, Theorem 4.12 Part II, p. 85].) Let now $\ell<s<1$, and note that $H^{s, p(s)}\left(\mathbb{R}^{N}\right) \hookrightarrow B_{\infty}^{s, p(s)}\left(\mathbb{R}^{N}\right)$, see [4, Theorem 6.2.4, p. 142]. Setting $m=1$, we have $m-1<\frac{N}{p(s)}<s<m$, and the result follows from the embedding $B_{\infty}^{s, p(s)}\left(\mathbb{R}^{N}\right) \hookrightarrow C^{0, \ell}\left(\overline{\mathbb{R}^{N}}\right)$. (See $[1$, Theorem 7.37, p. 233].)

We denote by $\left(e^{t \Delta}\right)_{t \geq 0}$ the heat semigroup on $\mathbb{R}^{N}$, and we recall that $e^{t \Delta}$ is a contraction on $L^{p}\left(\mathbb{R}^{N}\right)$ for all $1 \leq p \leq \infty$. Using (1.13), it follows immediately that $\left(e^{t \Delta}\right)_{t \geq 0}$ is also a contraction semigroup on $H^{s, p}\left(\mathbb{R}^{N}\right)$ for all $s \geq 0$ and $1<p<\infty$.

\section{Spatial Singularities AND ODEs}

In this section we study how a certain class of ordinary differential equations lead to loss of regularity. More precisely, we consider equations (1.9) and (1.10), which are ODEs with respect to time, as acting on functions depending on a space variable $|x| \leq 1$. In particular, the initial value $w(0, \cdot)=w_{0}(\cdot)$ is a function $w_{0}$ : $[-1,1] \rightarrow \mathbb{C}$. We wish to study the spatial regularity of $w(t, \cdot)$ as compared to the spatial regularity of $w_{0}$. This is a different phenomenon than finite-time blowup. For example, consider the ODE-initial value problem

$$
\left\{\begin{array}{l}
w_{t}=\lambda|w|^{\alpha} w \\
w(0, x)=x
\end{array}\right.
$$

with $\lambda \in \mathbb{C}, \lambda \neq 0$ and $|x| \leq 1$. If $\Re \lambda \neq 0$, then the solution of (2.1) is given by

It follows that

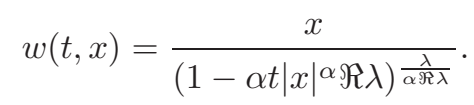

$$
\begin{gathered}
w_{x}(t, x)=\left(1+i \alpha t|x|^{\alpha} \Im \lambda\right)\left(1-\alpha t|x|^{\alpha} \Re \lambda\right)^{-\frac{\lambda+\alpha \Re \lambda}{\alpha \Re \lambda}} \\
w_{x x}(t, x)=\alpha t \frac{|x|^{\alpha}}{x}\left(1-\alpha t|x|^{\alpha} \Re \lambda\right)^{-\frac{\lambda+2 \alpha \Re \lambda}{\alpha}}\left[\lambda+\alpha \Re \lambda+i \alpha(\Re \lambda)\left(1+\lambda t|x|^{\alpha}\right)\right]
\end{gathered}
$$


for $x \neq 0$, as long as these formulas make sense. If $\Re \lambda=0$, then the solution of (2.1) is given by

$$
w(t, x)=\exp \left(i t|x|^{\alpha} \Im \lambda\right) w_{0}(x)
$$

and so,

$$
\begin{gathered}
w_{x}(t, x)=\left(1+i \alpha t|x|^{\alpha} \Im \lambda\right) e^{i t|x|^{\alpha} \Im \lambda} \\
w_{x x}(t, x)=i \alpha t(\Im \lambda)\left(1+\alpha+i \alpha t|x|^{\alpha} \Im \lambda\right) \frac{|x|^{\alpha}}{x} e^{i t|x|^{\alpha} \Im \lambda}
\end{gathered}
$$

for $t \geq 0$ and $x \neq 0$. In both cases, $w(t)$ is $C^{1}$ in $[-1,1]$ as long as it exists. However, if $\alpha<1$, we see that $w(t)$ fails to be twice differentiable at $x=0$, for $t>0$.

Somewhat surprisingly, it turns out that this loss of spatial regularity also occurs for regular perturbations of (2.1), as the following theorem shows.

Theorem 2.1. Let $0<\alpha<1, \lambda \in \mathbb{C} \backslash\{0\}, T>0, w_{0} \in C^{2}([-1,1], \mathbb{C})$ and $h \in C([0, T] \times[-1,1], \mathbb{C})$ such that $\partial_{y} h \in C([0, T] \times[-1,1], \mathbb{C})$. Suppose further that $w_{0}(0)=0$ and $h(t, 0)=0$ for $0 \leq t \leq T$. By possibly assuming that $T>0$ is smaller, it follows that there exists a solution $w \in C^{1}([0, T] \times[-1,1], \mathbb{C})$ of the equation

$$
\begin{cases}w_{t}=\lambda|w|^{\alpha} w+h & 0 \leq t \leq T,-1 \leq y \leq 1 \\ w(0, y)=w_{0}(y) & -1 \leq y \leq 1\end{cases}
$$

If $w_{0}^{\prime}(0) \neq 0$ and

$$
\int_{0}^{\tau} \sup _{0<|y| \leq 1} \frac{\left|\partial_{y} h(t, y)-\partial_{y} h(t, 0)\right|}{|y|^{\beta}}<\infty
$$

for some $0<\tau \leq T$ and $\beta>\alpha$, then

$$
\liminf _{|y| \downarrow 0} \frac{\left|\partial_{y} w(t, y)-\partial_{y} w(t, 0)\right|}{|y|^{\alpha}}>0
$$

for all sufficiently small $t>0$. In particular, $w(t, \cdot)$ is not twice differentiable at $y=0$ for any sufficiently small $0<t \leq T$.

Proof. The existence of the solution $w$ is straightforward, and

$$
w(t, 0)=0
$$

for all $0 \leq t \leq T$. For the rest of the proof, we consider for simplicity $0 \leq y \leq 1$, the extension to $-1 \leq y \leq 0$ will be clear. Set $f(t, y)=\partial_{y} h(t, y)$ and $v(t, y)=\partial_{y} w(t, y)$, so that $v, v_{t}, f \in C([0, T] \times[0,1])$. Differentiating equation (2.2) with respect to $y$ yields

$$
v_{t}=\lambda \frac{\alpha+2}{2}|w|^{\alpha} v+\lambda \frac{\alpha}{2}|w|^{\alpha-2} w^{2} \bar{v}+f
$$

pointwise in $[0, T] \times[0,1]$. Integrating $(2.6)$ and setting

$$
g=\lambda \frac{\alpha}{2}|w|^{\alpha-2} w^{2} \bar{v}+f
$$

we obtain

$$
v(t, y)=e^{A(t, y)} w_{0}^{\prime}(y)+\int_{0}^{t} e^{A(t, y)-A(s, y)} g(s, y) d s,
$$

pointwise on $[0, T] \times[0,1]$, where

$$
A(t, y)=\lambda \frac{\alpha+2}{2} \int_{0}^{t}|w(\sigma, y)|^{\alpha} d \sigma .
$$


We note that $A(t, 0)=0$ by $(2.5)$ so that

$$
v(t, 0)=w_{0}^{\prime}(0)+\int_{0}^{t} g(s, 0) d s,
$$

for all $0 \leq t \leq T$. Given $y>0$, it follows from (2.8) and (2.10) that

$$
\begin{aligned}
& v(t, y)-v(t, 0)=e^{A(t, y)}\left[w_{0}^{\prime}(y)-w_{0}^{\prime}(0)\right]+\left[e^{A(t, y)}-1\right] w_{0}^{\prime}(0) \\
& \quad+\int_{0}^{t}\left(e^{A(t, y)-A(s, y)}[g(s, y)-g(s, 0)]+\left[e^{A(t, y)-A(s, y)}-1\right] g(s, 0)\right) d s .
\end{aligned}
$$

Observe that, by assumption, $w_{0}(0)=0$ and

$$
z \stackrel{\text { def }}{=} w_{0}^{\prime}(0) \neq 0 .
$$

Thus for every $\varepsilon \in(0,1)$ there exists $0<\delta(\varepsilon) \leq \min \{T, \varepsilon\}$ such that

$$
|v(t, y)-z| \leq \varepsilon
$$

in the region

$$
\Delta_{\varepsilon}=\{(t, y) \in[0, T] \times[0,1] ; 0 \leq t \leq \delta(\varepsilon), 0 \leq y \leq \delta(\varepsilon)\} .
$$

Estimates (2.5) and (2.13) yield

$$
|w(t, y)-z y| \leq \varepsilon y
$$

in $\Delta_{\varepsilon}$. Next, we observe that by (2.9), the inequality $\left.|| z_{1}\right|^{\alpha}-\left|z_{2}\right|^{\alpha}|\leq| z_{1}-\left.z_{2}\right|^{\alpha}$ and (2.15)

$$
\begin{aligned}
\left.\left|A(t, y)-\lambda \frac{\alpha+2}{2} t\right| z\right|^{\alpha} y^{\alpha} \mid & \leq\left.|\lambda| \frac{\alpha+2}{2} \int_{0}^{t}|| w(\sigma, y)\right|^{\alpha}-|y z|^{\alpha} \mid d \sigma \\
& \leq|\lambda| \frac{\alpha+2}{2} \int_{0}^{t}|w(\sigma, y)-y z|^{\alpha} d \sigma \\
& \leq|\lambda| \frac{\alpha+2}{2} t \varepsilon^{\alpha} y^{\alpha}
\end{aligned}
$$

in $\Delta_{\varepsilon}$. In particular, $A$ is bounded. Since $w_{0}$ is $C^{2}$, it follows that

$$
\left|e^{A(t, y)}\left[w_{0}^{\prime}(y)-w_{0}^{\prime}(0)\right]\right| \leq C y
$$

in $\Delta_{\varepsilon}$. Moreover,

$$
\left|e^{A(t, y)}-1-A(t, y)\right| \leq C|A(t, y)|^{2} \leq C t^{2} y^{2 \alpha}
$$

in $\Delta_{\varepsilon}$, where we used (2.16) in the last inequality. It follows from (2.18) and (2.16) that

$$
\left|\left[e^{A(t, y)}-1\right] w_{0}^{\prime}(0)\right| \geq|\lambda| \frac{\alpha+2}{2}|z|\left(|z|^{\alpha}-\varepsilon^{\alpha}\right) t y^{\alpha}-C t^{2} y^{2 \alpha} .
$$

Next, using again the boundedness of $A$ in $\Delta_{\varepsilon}$, we deduce from (2.3) that

$$
\left|\int_{0}^{t} e^{A(t, y)-A(s, y)}[f(s, y)-f(s, 0)]\right| \leq C y^{\beta}
$$

in $\Delta_{\varepsilon}$. Using (2.16), we see that

$$
\left|e^{A(t, y)-A(s, y)}\right| \leq 1+C t y^{\alpha}
$$

for $0<s<t$ and $(t, y) \in \Delta_{\varepsilon}$. Moreover, it follows from (2.13) and (2.15) that

$$
\left.|| w\right|^{\alpha-2} w^{2} \bar{v} \mid \leq(|z|+\varepsilon)^{\alpha+1} y^{\alpha}
$$


in $\Delta_{\varepsilon}$. We deduce from $(2.7),(2.20),(2.21)$ and $(2.22)$ that

$$
\begin{aligned}
\left|\int_{0}^{t} e^{A(t, y)-A(s, y)}[g(s, y)-g(s, 0)]\right| & \leq C y^{\beta} \\
& +|\lambda| \frac{\alpha}{2}\left(1+C t y^{\alpha}\right)(|z|+\varepsilon)^{\alpha+1} t y^{\alpha} .
\end{aligned}
$$

Next,

$$
\begin{aligned}
\left|e^{A(t, y)-A(s, y)}-1\right| & \leq C|A(t, y)-A(s, y)| \leq C \int_{s}^{t}|w(\sigma, 0, y)|^{\alpha} \\
& \leq C(t-s)|y|^{\alpha}
\end{aligned}
$$

where we used (2.15) in the last inequality. Since $g$ is bounded, it follows that

$$
\left|\int_{0}^{t}\left[e^{A(t, y)-A(s, y)}-1\right] g(s, 0) d s\right| \leq C t^{2} y^{\alpha} .
$$

Finally, we observe that the various terms in the right-hand side of (2.11) are estimated by (2.17), (2.19), (2.23) and (2.24), and we deduce that

$$
\begin{aligned}
\frac{|v(t, y)-v(t, 0)|}{y^{\alpha}} & \geq|\lambda| \frac{\alpha+2}{2}|z|\left(|z|^{\alpha}-\varepsilon^{\alpha}\right) t-C t^{2} y^{\alpha}-C y-C y^{\beta-\alpha} \\
& -|\lambda| \frac{\alpha}{2}\left(1+C t y^{\alpha}\right)(|z|+\varepsilon)^{\alpha+1} t-C t^{2}
\end{aligned}
$$

in $\Delta_{\varepsilon}$. It follows that

$$
\liminf _{y \downarrow 0} \frac{|v(t, y)-v(t, 0)|}{y^{\alpha}} \geq t|\lambda|\left(\frac{\alpha+2}{2}|z|\left(|z|^{\alpha}-\varepsilon^{\alpha}\right)-\mid \frac{\alpha}{2}(|z|+\varepsilon)^{\alpha+1}\right)-C t^{2} .
$$

Choosing $\varepsilon>0$ and $t>0$ sufficiently small, we see that

$$
\liminf _{y \downarrow 0} \frac{|v(t, y)-v(t, 0)|}{y^{\alpha}} \geq t|\lambda| \frac{|z|^{\alpha+1}}{2}
$$

from which estimate (2.4) follows.

Remark 2.2. The assumption that $w_{0} \in C^{2}([0,1], \mathbb{C})$ is used only once in the proof, see (2.17). It could be replaced by the weaker condition $w_{0} \in C^{1, \mu}([0,1], \mathbb{C})$ with $\alpha<\mu<1$.

\section{Semilinear equations as perturbations of an ODE}

In this section we show that Theorem 2.1 easily implies Theorems 1.1 and 1.5.

Proof of Theorem 1.1. We recall that if $\Delta u_{0} \in C_{0}\left(\mathbb{R}^{N}\right), u_{0} \in C^{3}\left(\mathbb{R}^{N}\right) \cap W^{3, \infty}\left(\mathbb{R}^{N}\right)$, and $\left|\partial^{\gamma} u\right|_{\alpha}<\infty$ for all multi-indices $\gamma$ such that $|\gamma|=3$, then $u$ is once continuously differentiable with respect to $t$, three times continuously differentiable with respect to $x, u_{t}$ is $\frac{\alpha}{2}$-Hölder continuous in $t$ and $\sup _{0 \leq t \leq T}\left|\partial^{\gamma} u(t)\right|_{\alpha}<\infty$ for $|\gamma|=3$ and $0<T<T_{\max }$. See Theorem A.1 below for a precise statement. We write the variable in $\mathbb{R}^{N}$ in the form $x=\left(x^{\prime}, y\right), x^{\prime} \in \mathbb{R}^{N-1}, y \in \mathbb{R}$. Accordingly, we write $u_{0}(x)=u_{0}\left(x^{\prime}, y\right)$ and $u(t, x)=u\left(t, x^{\prime}, y\right)$.

Let $u_{0} \in C_{0}\left(\mathbb{R}^{N}\right)$ with $\Delta u_{0} \in C_{0}\left(\mathbb{R}^{N}\right)$. Suppose further that $u_{0}\left(x^{\prime},-y\right)=$ $-u_{0}\left(x^{\prime}, y\right)$ for all $x^{\prime} \in \mathbb{R}^{N-1}$ and $y \in \mathbb{R}$, and $\partial_{y} u_{0}(0,0) \neq 0$. Note that $u$ inherits the anti-symmetry of the initial condition, i.e. $u\left(t, x^{\prime},-y\right) \equiv-u\left(t, x^{\prime}, y\right)$ for all $0<t<T_{\max }$. Moreover, there exists $0<t_{0}<T_{\max }$ such that $\partial_{y} u(t, 0,0) \neq 0$ for all $0 \leq t \leq t_{0}$. Thus we see that for all $0 \leq t \leq t_{0}, u(t)$ satisfies the same 
assumptions as $u_{0}$. Therefore, it suffices to prove (1.8) for $s=0$. Assume by contradiction that

$$
\int_{0}^{T}\left|\partial_{y} \Delta u(t)\right|_{\beta}<\infty
$$

for some $\alpha<\beta \leq 1$ and $0<T<t_{0}$. We apply Theorem 2.1 with $w(t, y)=$ $u(t, 0, y)$ and $h(t, y)=\Delta u(t, 0, y)$. The anti-symmetry property of $u$ implies that $w(t, 0)=h(t, 0)=0$ for all $0 \leq t<T_{\max }$. Moreover, it follows from (3.1) that the assumption (2.3) is satisfied. Therefore, we deduce from (2.4) that if $t_{0}>0$ is sufficiently small, then

$$
\limsup _{|y| \rightarrow 0} \frac{\left|\partial_{y} w(t, y)-\partial_{y} w(t, 0)\right|}{|y|^{\alpha}}>0
$$

for $0<t<t_{0}$. Since $\partial_{y} w(t, y)=\partial_{y} u(t, 0, y)$ is $C^{1}$ in $y$, this yields a contradiction. The result follows, since we can choose $u_{0} \in C_{\mathrm{c}}^{\infty}\left(\mathbb{R}^{N}\right)$ as above.

Remark 3.1. If $\lambda \in \mathbb{R} \backslash\{0\}$, then the statement of Theorem 1.1 can be improved in the sense that there exists an initial value $u_{0} \in C_{\mathrm{c}}^{\infty}\left(\mathbb{R}^{N}\right)$ for which (1.8) holds for all $0 \leq s<t<T_{\max }$. (We do not require that $t$ is small.) Indeed, let $u_{0} \in C_{0}\left(\mathbb{R}^{N}\right)$ with $\Delta u_{0} \in C_{0}\left(\mathbb{R}^{N}\right)$. Suppose further that $u_{0}\left(x^{\prime},-y\right)=-u_{0}\left(x^{\prime}, y\right)$ for all $x^{\prime} \in \mathbb{R}^{N-1}$ and $y \in \mathbb{R}, u_{0}\left(x^{\prime}, y\right) \geq 0$ for all $x^{\prime} \in \mathbb{R}^{N-1}$ and $y>0$, and $\partial_{y} u_{0}(0,0)>0$. Since $u$ inherits the anti-symmetry of the initial condition, i.e. $u\left(t, x^{\prime},-y\right) \equiv-u\left(t, x^{\prime}, y\right)$, it follows that, restricted to the open half space $\mathbb{R}_{+}^{N}=\mathbb{R}^{N-1} \times(0, \infty), u$ is a solution of the Dirichlet initial value problem on $\mathbb{R}_{+}^{N}$. In particular, $u\left(t, x^{\prime}, y\right)>0$ for $y>0$, and $\partial_{y} u\left(t, x^{\prime}, 0\right)>0$. Thus we see that for all $0 \leq t<T_{\max }, u(t)$ satisfies the same assumptions as $u_{0}$, and we can conclude as above.

Ne turn next to Theorem 1.5. Equation (1.2) is a particular case of the following nonlinear complex Ginzburg-Landau equation

$$
\left\{\begin{array}{l}
u_{t}=e^{i \theta} \Delta u+\lambda|u|^{\alpha} u \\
u(0)=u_{0}
\end{array}\right.
$$

in $\mathbb{R}^{N}$, where $-\frac{\pi}{2} \leq \theta \leq \frac{\pi}{2}$, and $0<\alpha<1$. Theorem 1.5 is therefore a consequence of the following result.

Theorem 3.2. Let $0<\lambda<1, \lambda \in \mathbb{C} \backslash\{0\}$ and $-\frac{\pi}{2} \leq \theta \leq \frac{\pi}{2}$. Suppose $s>3+\frac{N}{2}+\alpha$. There exists $u_{0} \in C_{\mathrm{c}}^{\infty}\left(\mathbb{R}^{N}\right)$ such that there is no $T>0$ for which there exists a solution $u \in C\left([0, T], H^{s}\left(\mathbb{R}^{N}\right)\right)$ of $(3.2)$.

Remark 3.3. (i) Suppose $s>3+\frac{N}{2}$ (so that $H^{s}\left(\mathbb{R}^{N}\right) \subset C^{3}\left(\mathbb{R}^{N}\right) \cap W^{3, \infty}\left(\mathbb{R}^{N}\right)$ ). If $u \in C\left([0, T], H^{s}\left(\mathbb{R}^{N}\right)\right)$, then $\Delta u,|u|^{\alpha} u \in C\left([0, T] \times \mathbb{R}^{N}\right) \cap C\left([0, T], L^{2}\left(\mathbb{R}^{N}\right)\right)$ and $\nabla \Delta u, \nabla\left(|u|^{\alpha} u\right) \in C\left([0, T] \times \mathbb{R}^{N}\right)$. Therefore, equation (3.2) makes sense for such a $u$. Furthermore, $u_{t} \in C\left([0, T] \times \mathbb{R}^{N}\right) \cap C\left([0, T], L^{2}\left(\mathbb{R}^{N}\right)\right)$ and $\nabla u_{t} \in C\left([0, T] \times \mathbb{R}^{N}\right)$.

(ii) It follows from the preceding observation that it makes sense to talk of a solution of $(3.2)$ in $C\left([0, T], H^{s}\left(\mathbb{R}^{N}\right)\right)$ if $s>3+\frac{N}{2}$. Such a solution satisfies the integral equation

$$
u(t)=e^{i t \Delta} u_{0}+i \lambda \int_{0}^{t} e^{i(t-s) \Delta}\left(|u|^{\alpha} u\right)(s) d s .
$$


Using the embedding $H^{s}\left(\mathbb{R}^{N}\right) \hookrightarrow L^{\infty}\left(\mathbb{R}^{N}\right)$, it follows easily that such a solution is unique. In particular, if $u_{0}$ is anti-symmetric in the last variable, then so is any solution $u \in C\left([0, T], H^{s}\left(\mathbb{R}^{N}\right)\right)$ of (3.2).

Proof of Theorem 3.2. We write the variable in $\mathbb{R}^{N}$ in the form $x=\left(x^{\prime}, y\right), x^{\prime} \in$ $\mathbb{R}^{N-1}, y \in \mathbb{R}$. Accordingly, we write $u_{0}(x)=u_{0}\left(x^{\prime}, y\right)$ and $u(t, x)=u\left(t, x^{\prime}, y\right)$.

Let $u_{0} \in C_{\mathrm{c}}^{\infty}\left(\mathbb{R}^{N}\right)$ and suppose that $u_{0}\left(x^{\prime},-y\right)=-u_{0}\left(x^{\prime}, y\right)$ for all $x^{\prime} \in \mathbb{R}^{N-1}$ and $y \in \mathbb{R}$, and $\partial_{y} u_{0}(0,0)>0$. Assume by contradiction that there exist $T>0$, $s>3+\frac{N}{2}+\alpha$, and a solution $u \in C\left([0, T], H^{s}\left(\mathbb{R}^{N}\right)\right)$ of (3.2). As observed in Remark 3.3 (ii), it follows in particular that $u\left(t, x^{\prime},-y\right) \equiv-u\left(t, x^{\prime}, y\right)$ for all $0 \leq$ $t \leq T$. We apply Theorem 2.1 with $w(t, y)=u(t, 0, y)$ and $h(t, y)=e^{i \theta} \Delta u(t, 0, y)$. The regularity assumptions on $w$ and $h$ are satisfied by Remark 3.3 (i). The antisymmetry property of $u$ imply that $w(t, 0)=h(t, 0)=0$ for all $0 \leq t<T_{\max }$. Moreover, it follows from Sobolev's embedding theorem (see (1.15)) that

$$
\left|\partial_{y} h(t, y)-\partial_{y} h(t, 0)\right| \leq C|y|^{s-3-\frac{N}{2}}
$$

for all $t \in[0, T]$ and $y \in \mathbb{R}$, so that the assumption (2.3) is satisfied. Therefore, we deduce from (2.4) that if $t_{0}>0$ is sufficiently small, then

$$
\limsup _{|y| \rightarrow 0} \frac{|v(t, y)-v(t, 0)|}{|y|^{\alpha}}>0
$$

for $0<t<t_{0}$. Since $v(t, y)=\partial_{y} u(t, 0, y)$ is $C^{1}$ in $y$ by Remark 3.3 (i), this yields a contradiction.

Remark 3.4. Observe that if $u_{0}$ is as in the proof of either Theorem 1.1 or Theorem 3.2 , then so is $\varepsilon u_{0}$ for all $\varepsilon \neq 0$.

\section{Time-Pointwise LACK of Regularity: The heAT EQUATion}

In this section we prove Theorems 1.7 and 1.10. As motivation for the proof of Theorem 1.7, consider an initial value $u_{0} \in C_{\mathrm{c}}^{\infty}\left(\mathbb{R}^{N}\right)$ which is odd with respect to the $N^{\text {th }}$ variable $y$ and such that $u_{0}\left(x^{\prime}, y\right)=C\left(x^{\prime}\right) y$ for small $|y|$, and write $\left|u\left(t, x^{\prime}, y\right)\right|^{\alpha} u\left(t, x^{\prime}, y\right)=C\left(x^{\prime}\right) \gamma(t)|y|^{\alpha} y+\widetilde{w}\left(t, x^{\prime}, y\right)$ where $\left|\widetilde{w}\left(t, x^{\prime}, y\right)\right| \leq C|y|^{\alpha+2}$. This decomposition makes it possible to explicitly calculate $\partial_{y}^{5}\left[e^{(t-s) \Delta}|u|^{\alpha} u\right]_{y=0}$. Incorporating this result into the integral (1.11) yields the desired property.

The following two lemmas show explicitly how these ideas are implemented.

Lemma 4.1. Let $\psi \in C(\mathbb{R})$ such that $|\psi(x)| \leq C\left(1+|x|^{m}\right)$ for some $m \geq 0$. Let $\sigma>0$ and set

$$
z=e^{\frac{\sigma}{4} \Delta} \psi \in C^{\infty}(\mathbb{R}) .
$$

It follows that

$$
\partial_{x}^{5} z(0)=8 \pi^{-\frac{1}{2}} \sigma^{-3} \int_{\mathbb{R}} e^{-y^{2}}\left[15-20 y^{2}+4 y^{4}\right](y \sqrt{\sigma}) \psi(y \sqrt{\sigma}) d y .
$$

Proof. We have

$$
z(x)=(\pi \sigma)^{-\frac{1}{2}} \int_{\mathbb{R}} e^{-\frac{(x-y)^{2}}{\sigma}} \psi(y) d y,
$$

so that

$$
\partial_{x}^{n} z(x)=(\pi \sigma)^{-\frac{1}{2}} \int_{\mathbb{R}} \partial_{x}^{n}\left(e^{-\frac{(x-y)^{2}}{\sigma}}\right) \psi(y) d y .
$$


T. CAZENAVE, F. DICKSTEIN, AND F. B. WEISSLER

We next calculate

$$
\partial_{x}^{5}\left(e^{-\frac{(x-y)^{2}}{\sigma}}\right)=-e^{-\frac{(x-y)^{2}}{\sigma}}\left[\frac{120(x-y)}{\sigma^{3}}-\frac{160(x-y)^{3}}{\sigma^{4}}+\frac{32(x-y)^{5}}{\sigma^{5}}\right],
$$

so that

$$
\partial_{x}^{5}\left(e^{-\frac{(x-y)^{2}}{\sigma}}\right)_{\mid x=0}=8 e^{-\frac{y^{2}}{\sigma}} \sigma^{-3} y\left[15-\frac{20 y^{2}}{\sigma}+\frac{4 y^{4}}{\sigma^{2}}\right] .
$$

Thus we deduce from (4.3) that

$$
\partial_{x}^{5} z(0)=8 \pi^{-\frac{1}{2}} \sigma^{-\frac{7}{2}} \int_{\mathbb{R}} e^{-\frac{y^{2}}{\sigma}}\left[15-\frac{20 y^{2}}{\sigma}+\frac{4 y^{4}}{\sigma^{2}}\right] y \psi(y) d y,
$$

from which (4.2) follows.

Lemma 4.2. If $\psi(x)=|x|^{\alpha} x$ with $\alpha>0$, then

$$
\partial_{x}^{5}\left[e^{\frac{\sigma}{4} \Delta} \psi\right]_{\mid x=0}=-C_{\alpha} \sigma^{-2+\frac{\alpha}{2}},
$$

for all $\sigma>0$, where

$$
C_{\alpha}=\pi^{-\frac{1}{2}} \frac{32 \alpha(2-\alpha)}{(\alpha+3)(\alpha+5)} \int_{\mathbb{R}} e^{-y^{2}}|y|^{\alpha+6} .
$$

Proof. Considering (4.2), we must calculate

$$
\begin{aligned}
\int_{\mathbb{R}} e^{-y^{2}}\left[15-20 y^{2}+4 y^{4}\right]|y \sqrt{\sigma}|^{\alpha+2} d y & \\
& =\sigma^{1+\frac{\alpha}{2}} \int_{\mathbb{R}} e^{-y^{2}}\left[15-20 y^{2}+4 y^{4}\right]|y|^{\alpha+2} d y .
\end{aligned}
$$

Note that, given any $\beta \geq 0$,

$$
\int_{\mathbb{R}} e^{-y^{2}}|y|^{\beta}=\int_{\mathbb{R}} e^{-y^{2}}\left(\frac{|y|^{\beta} y}{\beta+1}\right)^{\prime}=\frac{2}{\beta+1} \int_{\mathbb{R}} e^{-y^{2}}|y|^{\beta+2} .
$$

It follows that

$$
\int_{\mathbb{R}} e^{-y^{2}}|y|^{\alpha+2}=\frac{2}{\alpha+3} \int_{\mathbb{R}} e^{-y^{2}}|y|^{\alpha+4}=\frac{4}{(\alpha+3)(\alpha+5)} \int_{\mathbb{R}} e^{-y^{2}}|y|^{\alpha+6},
$$

and

Therefore,

$$
\int_{\mathbb{R}} e^{-y^{2}}|y|^{\alpha+4}=\frac{2}{\alpha+5} \int_{\mathbb{R}} e^{-y^{2}}|y|^{\alpha+6} .
$$

$$
\int_{\mathbb{R}} e^{-y^{2}}\left[15-20 y^{2}+4 y^{4}\right]|y|^{\alpha+2} d y=\frac{4 \alpha(\alpha-2)}{(\alpha+3)(\alpha+5)} \int_{\mathbb{R}} e^{-y^{2}}|y|^{\alpha+6}
$$

The result now follows from (4.2), (4.8) and (4.9).

Proof of Theorem 1.7. We write the variable in $\mathbb{R}^{N}$ in the form $x=\left(x^{\prime}, y\right), x^{\prime} \in$ $\mathbb{R}^{N-1}, y \in \mathbb{R}$. Accordingly, we write $u_{0}(x)=u_{0}\left(x^{\prime}, y\right)$ and $u(t, x)=u\left(t, x^{\prime}, y\right)$. We note that

$$
e^{t \Delta}=e^{t \Delta \Delta_{x^{\prime}}} e^{t \partial_{y}^{2}}
$$

where $e^{t \Delta_{x^{\prime}}}$ is the convolution in $\mathbb{R}^{N-1}$ with the kernel $(4 \pi t)^{-\frac{N-1}{2}} e^{-\frac{\left|x^{\prime}\right|^{2}}{4 t}}$ and $e^{t \partial_{y}^{2}}$ is the convolution in $\mathbb{R}$ with the kernel $(4 \pi t)^{-\frac{1}{2}} e^{-\frac{y^{2}}{4 t}}$.

Let $u_{0} \in C_{\mathrm{c}}^{\infty}\left(\mathbb{R}^{N}\right)$ and let $u \in C\left(\left[0, T_{\max }\right), C_{0}(\mathbb{R}) \cap L^{1}\left(\mathbb{R}^{N}\right)\right)$ be the corresponding maximal solution of (1.1). Assume that

$$
u_{0}\left(x^{\prime},-y\right) \equiv-u_{0}\left(x^{\prime}, y\right) \text { and } \partial_{y} u_{0}(0,0) \neq 0 .
$$


Recall that $u$ is $C^{1}$ in time and $C^{3}$ in space, and that $\sup _{0 \leq t \leq T}\|u(t)\|_{W^{2, \infty}}<\infty$ for all $0<T<T_{\max }$. (See e.g. Theorem A.1.) Under the assumption (4.11) it follows that for all $0 \leq t \leq T$ and $x^{\prime} \in \mathbb{R}^{N-1}, u\left(t, x^{\prime}, \cdot\right)$ is odd and $u\left(t, x^{\prime}, y\right)>0$ for $y>0$. Moreover, if we set

$$
\begin{gathered}
\eta_{0}=\partial_{y} u_{0}(0,0) \\
\eta\left(t, x^{\prime}\right)=\partial_{y} u\left(t, x^{\prime}, 0\right), \quad 0 \leq t \leq T, x^{\prime} \in \mathbb{R}^{N-1}
\end{gathered}
$$

then $\eta \in C\left(\left[0, T_{\max }\right) \times \mathbb{R}^{N-1}\right)$. Therefore, it follows from (4.11) that for every $0<\varepsilon<1$ there exists $0<\delta_{\varepsilon}<T_{\max }$ such that

$$
\sup _{\substack{0 \leq s \leq \delta_{\varepsilon} \\\left|x^{\prime}\right| \leq \delta_{\varepsilon}}}\left|\eta\left(s, x^{\prime}\right)-\eta_{0}\right| \leq \varepsilon\left|\eta_{0}\right| \text {. }
$$

Let $w$ be defined by

$$
u\left(s, x^{\prime}, y\right)=\eta\left(s, x^{\prime}\right) y+w\left(s, x^{\prime}, y\right) .
$$

We claim that

$$
\left|w\left(s, x^{\prime}, y\right)\right| \leq C y^{2},
$$

for all $s \in[0, T], x^{\prime} \in \mathbb{R}^{N-1}$ and $y \in \mathbb{R}$, where $C=\frac{1}{2} \sup _{0 \leq t \leq T}\|u(t)\|_{W^{2, \infty}}$. Indeed, fix $0 \leq s \leq T$ and $x^{\prime} \in \mathbb{R}^{N-1}$, and set $h(y)=w\left(s, x^{\prime}, y\right)=u\left(s, x^{\prime}, y\right)-\eta\left(s, x^{\prime}\right) y$. We have $h^{\prime}(y)=\partial_{y} u\left(s, x^{\prime}, y\right)-\eta\left(s, x^{\prime}\right)$ and $h^{\prime \prime}(y)=\partial_{y}^{2} u\left(s, x^{\prime}, y\right)$. In particular, $h(0)=h^{\prime}(0)=0$ so that

$$
h(y)=\int_{0}^{y} \int_{0}^{\tau} \partial_{y}^{2} u\left(s, x^{\prime}, \sigma\right) d \sigma d \tau
$$

and so

$$
\left|w\left(s, x^{\prime}, y\right)\right|=|h(y)| \leq \frac{1}{2} y^{2}\|u(s, \cdot, \cdot)\|_{W^{2, \infty}}
$$

which proves (4.16). Since $u$ is bounded, we deduce easily from (4.15)-(4.16) that

$$
\left[|u|^{\alpha} u\right]\left(s, x^{\prime}, y\right)=\left|\eta\left(s, x^{\prime}\right) y\right|^{\alpha} \eta\left(s, x^{\prime}\right) y+\widetilde{w}\left(s, x^{\prime}, y\right)
$$

with

$$
\left|\widetilde{w}\left(s, x^{\prime}, y\right)\right| \leq C|y|^{\alpha+2},
$$

for all $s \in[0, T]$ and $\left(x^{\prime}, y\right) \in \mathbb{R}^{N}$. It follows from Lemma 4.2 that if $\psi(y)=|y|^{\alpha} y$, then

$$
\partial_{y}^{5}\left[e^{\frac{\sigma}{4} \partial_{y}^{2}} \psi\right]_{\mid y=0}=-C_{\alpha} \sigma^{-2+\frac{\alpha}{2}}
$$

for all $\sigma>0$, where $C_{\alpha}>0$ is given by (4.7). We deduce from (4.10) and (4.19) that

$$
\begin{aligned}
\partial_{y}^{5}\left[e^{(t-s) \Delta}\left(\left|\eta\left(s, x^{\prime}\right) y\right|^{\alpha} \eta\left(s, x^{\prime}\right) y\right)\right]_{\mid y=0} \\
\quad=-C_{\alpha}(4(t-s))^{-2+\frac{\alpha}{2}} e^{(t-s) \Delta_{x^{\prime}}}\left[\left|\eta\left(s, x^{\prime}\right)\right|^{\alpha} \eta\left(s, x^{\prime}\right)\right] .
\end{aligned}
$$

On the other hand, it follows from (4.14) that there exists $C$ independent of $0<$ $\varepsilon<1$ such that

$$
\left.\sup _{\substack{0 \leq s \leq \delta_{\varepsilon} \\\left|x^{\prime}\right| \leq \delta_{\varepsilon}}}|| \eta\left(s, x^{\prime}\right)\right|^{\alpha} \eta\left(s, x^{\prime}\right)-\left|\eta_{0}\right|^{\alpha} \eta_{0} \mid \leq \varepsilon C .
$$

By possibly choosing $\delta_{\varepsilon}>0$ smaller, we deduce that

$$
\left.\sup _{\substack{0 \leq s<t \leq \delta_{\varepsilon} \\\left|x^{\prime}\right| \leq \delta_{\varepsilon}}}\left|e^{(t-s) \Delta_{x^{\prime}}}\left[\left|\eta\left(s, x^{\prime}\right)\right|^{\alpha} \eta\left(s, x^{\prime}\right)\right]-\right| \eta_{0}\right|^{\alpha} \eta_{0} \mid \leq \varepsilon C .
$$


It follows from (4.20) and (4.21) that

$$
\begin{array}{r}
\left.\mid \partial_{y}^{5}\left[e^{(t-s) \Delta}\left|\eta\left(s, x^{\prime}\right) y\right|^{\alpha} \eta\left(s, x^{\prime}\right) y\right)\right]_{\mid y=0}+C_{\alpha}(4(t-s))^{-2+\frac{\alpha}{2}}\left|\eta_{0}\right|^{\alpha} \eta_{0} \mid \\
\leq \varepsilon C(t-s)^{-2+\frac{\alpha}{2}}
\end{array}
$$

for all $0 \leq s<t \leq \delta_{\varepsilon}$ and $\left|x^{\prime}\right| \leq \delta_{\varepsilon}$. On the other hand, it follows from (4.2) that

$$
\begin{array}{r}
\left|\partial_{y}^{5}\left[e^{(t-s) \Delta} \widetilde{w}\left(s, x^{\prime}, y\right)\right]_{\mid y=0}\right|=\left|e^{(t-s) \Delta_{x^{\prime}}} \partial_{y}^{5}\left[e^{(t-s) \partial_{y}^{2}} \widetilde{w}\left(s, x^{\prime}, y\right)\right]_{\mid y=0}\right| \\
\leq C(t-s)^{-\frac{5}{2}} e^{(t-s) \Delta_{x^{\prime}}}\left(\int_{\mathbb{R}} e^{-y^{2}}\left|15-20 y^{2}+4 y^{4}\right||y|\left|\widetilde{w}\left(s, x^{\prime}, y \sqrt{4(t-s)}\right)\right| d y\right) \\
\leq C(t-s)^{-\frac{5}{2}} e^{(t-s) \Delta_{x^{\prime}}}\left(\int_{\mathbb{R}} e^{-\frac{y^{2}}{2}}\left|\widetilde{w}\left(s, x^{\prime}, y \sqrt{4(t-s)}\right)\right| d y\right) .
\end{array}
$$

Applying (4.18), we deduce that

$$
\left|\partial_{y}^{5}\left[e^{(t-s) \Delta} \widetilde{w}\left(s, x^{\prime}, y\right)\right]_{\mid y=0}\right| \leq C(t-s)^{-\frac{3}{2}+\frac{\alpha}{2}} \int_{\mathbb{R}} e^{-\frac{y^{2}}{2}}|y|^{\alpha+2} \leq C(t-s)^{-\frac{3}{2}+\frac{\alpha}{2}} .
$$

It now follows from $(4.17),(4.22)$ and (4.23) that

$$
\begin{aligned}
\mid \partial_{y}^{5}\left[e^{(t-s) \Delta}\left|u\left(s, x^{\prime}, y\right)\right|^{\alpha} u\left(s, x^{\prime}, y\right)\right]_{\mid y=0} \\
+C_{\alpha}(4(t-s))^{-2+\frac{\alpha}{2}}\left|\eta_{0}\right|^{\alpha} \eta_{0} \mid \leq \varepsilon C(t-s)^{-2+\frac{\alpha}{2}}
\end{aligned}
$$

for all $0 \leq s<t \leq \delta_{\varepsilon}$ and $\left|x^{\prime}\right| \leq \delta_{\varepsilon}$.

The point is that $(t-s)^{-2+\frac{\alpha}{2}}$ is not integrable in $s$ at $s=t$ under the assumption $0<\alpha<2$. We now conclude the proof as follows. Fix $0<\tau<T_{\max }$ and, given any $0 \leq t<\tau$, set

$$
\mathcal{I}\left(t, \tau, x^{\prime}, y\right)=\int_{0}^{t} e^{(\tau-s) \Delta}|u(s)|^{\alpha} u(s) d s .
$$

Since $\tau-s \geq \tau-t>0$ for $s \in[0, t]$, the smoothing effect of the heat semigroup implies that the integrand in (4.25) is integrable as a function with values in $H^{m}\left(\mathbb{R}^{N}\right)$ for all $m \geq 0$. Choosing $m$ large enough so that $H^{m}\left(\mathbb{R}^{N}\right) \subset C^{5}\left(\mathbb{R}^{N}\right)$, we see that the formula

$$
\partial_{y}^{5} \mathcal{I}\left(t, \tau, x^{\prime}, 0\right)=\int_{0}^{t} \partial_{y}^{5}\left[e^{(\tau-s) \Delta}|u(s)|^{\alpha} u(s)\right]_{\mid y=0} d s
$$

makes sense. Applying (4.24) with $t$ replaced by $\tau$, we deduce that

$$
\begin{aligned}
\left|\partial_{y}^{5} \mathcal{I}\left(t, \tau, x^{\prime}, 0\right)\right| & \geq\left[C_{\alpha} 4^{-2+\frac{\alpha}{2}}\left|\eta_{0}\right|^{\alpha+1}-\varepsilon C\right] \int_{0}^{t}(\tau-s)^{-2+\frac{\alpha}{2}} d s \\
& =\frac{2}{2-\alpha}\left[C_{\alpha} 4^{-2+\frac{\alpha}{2}}\left|\eta_{0}\right|^{\alpha+1}-\varepsilon C\right]\left[(\tau-t)^{-\frac{2-\alpha}{2}}-\tau^{-\frac{2-\alpha}{2}}\right]
\end{aligned}
$$

for $0<t<\tau \leq \delta_{\varepsilon}$ and $\left|x^{\prime}\right| \leq \delta_{\varepsilon}$. We now fix $s, p$ such that $s \geq 5+\frac{1}{p}$ and $\varepsilon$ sufficiently small so that $C_{\alpha} 4^{-2+\frac{\alpha}{2}}\left|\eta_{0}\right|^{\alpha+1}>\varepsilon C$, and we deduce from (4.27) and the embedding $H^{s, p}(\mathbb{R}) \hookrightarrow W^{5, \infty}(\mathbb{R})$ that

$$
\left\|\mathcal{I}\left(t, \tau, x^{\prime}, \cdot\right)\right\|_{H^{s, p}(\mathbb{R})} \geq a(\tau-t)^{-1+\frac{\alpha}{2}}-a t^{-1+\frac{\alpha}{2}}-A,
$$

for some constants $a, A>0$ independent of $0<t<\tau \leq \delta_{\varepsilon}$ and $\left|x^{\prime}\right| \leq \delta_{\varepsilon}$. We now use the property

$$
\|u\|_{L_{x^{\prime}}^{p}\left(\left\{\left|x^{\prime}\right|<\delta_{\varepsilon}\right\}, H^{s, p}\left(\mathbb{R}_{y}\right)\right)} \leq\|u\|_{L_{x^{\prime}}^{p}\left(\mathbb{R}^{N-1}, H^{s, p}\left(\mathbb{R}_{y}\right)\right)} \leq C\|u\|_{H^{s, p}\left(\mathbb{R}_{x^{\prime}, y}^{N}\right)},
$$


(see (1.14)), and we deduce from (4.28) that for some constant $\nu>0$

$$
\nu\|\mathcal{I}(t, \tau)\|_{H^{s, p}\left(\mathbb{R}^{N}\right)} \geq a(\tau-t)^{-1+\frac{\alpha}{2}}-a t^{-1+\frac{\alpha}{2}}-A
$$

so that

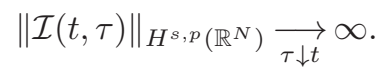

Observe that

$$
\begin{aligned}
\lambda \mathcal{I}\left(t, \tau, x^{\prime}, y\right) & =\lambda e^{(\tau-t) \Delta} \int_{0}^{t} e^{(t-s) \Delta}|u(s)|^{\alpha} u(s) d s \\
& =e^{(\tau-t) \Delta}\left[u(t)-e^{t \Delta} u_{0}\right]
\end{aligned}
$$

hence

$$
|\lambda|\|\mathcal{I}(t, \tau)\|_{H^{s, p}} \leq\|u(t)\|_{H^{s, p}}+\left\|u_{0}\right\|_{H^{s, p}} .
$$

Note that $\left\|u_{0}\right\|_{H^{s, p}}<\infty$. Therefore, letting $\tau \downarrow t$ and applying (4.30), we conclude that $\|u(t)\|_{H^{s, p}}=\infty$.

Remark 4.3. Observe that if $u_{0}$ is as in the proof of Theorem 1.7, then so is $\varepsilon u_{0}$ for all $\varepsilon \neq 0$.

Remark 4.4. A careful analysis of the proof of Theorem 1.7 shows that the property $C_{\alpha}>0$ (i.e., $\alpha<2$ ), where $C_{\alpha}$ is given by (4.7), could in principle be replaced by the condition $C_{\alpha} \neq 0$ (i.e., $\alpha \neq 2$ ). On the other hand, the condition $\alpha<2$ is crucial in proving (4.30) by letting $\tau \downarrow t$ in (4.29).

Proof of Theorem 1.10. We argue by contradiction. Suppose that for some $s \geq 0$ the Cauchy problem (1.1) is locally well posed for small data in $H^{s}\left(\mathbb{R}^{N}\right)$. Using Theorem 1.7 and Remark 4.3 with $p=2$ we see that $2 s \leq 11$. It follows then from (1.12) that

$$
N-2 s>\frac{4}{\alpha}
$$

A scaling argument allows us now to conclude. Indeed, let $\varphi \in C_{\mathrm{c}}^{\infty}\left(\mathbb{R}^{N}\right), \varphi \neq 0$. Since $\lambda>0$, for $k>0$ sufficiently large the (classical) solution $u$ of (1.1) with initial value $u_{0}=k \varphi$ blows up in finite time, say at $T_{\max }$. Given $\mu>0$, let

$$
u_{\mu}(t, x)=\mu^{\frac{2}{\alpha}} u\left(\mu^{2} t, \mu x\right)
$$

It follows that $u_{\mu}$ is a solution of (1.1) with the initial value

$$
u_{0}^{\mu}(x)=\mu^{\frac{2}{\alpha}} u_{0}(\mu x),
$$

which blows up at

$$
T_{\max }^{\mu}=\frac{T_{\max }}{\mu^{2}} \underset{\mu \rightarrow \infty}{\longrightarrow} 0 .
$$

On the other hand,

$$
\left\|u_{0}^{\mu}\right\|_{H^{s}} \leq \mu^{\frac{2}{\alpha}+s-\frac{N}{2}}\left\|u_{0}\right\|_{H^{s}}
$$

for $\mu \geq 1$. Using (4.31), we see that

$$
\left\|u_{0}^{\mu}\right\|_{H^{s}} \underset{\mu \rightarrow \infty}{\longrightarrow} 0 .
$$

Comparing (4.36) and (4.34), we conclude that (1.1) cannot be locally well posed for small data in $H^{s}\left(\mathbb{R}^{N}\right)$. 


\section{ApPENDix A. HöLDER REgUlARITy FOR THE HEAT EQUATION}

In this section, we state a classical regularity result for the heat equation (1.1). For completeness, we give the proof, which is based on classical arguments.

Theorem A.1. Let $\alpha>0, \lambda \in \mathbb{C}, u_{0} \in C_{0}\left(\mathbb{R}^{N}\right)$ and let $u \in C\left(\left[0, T_{\max }\right), C_{0}\left(\mathbb{R}^{N}\right)\right)$ be the corresponding maximal solution of (1.1). Fix $0<\widetilde{\alpha}<1$ with $\widetilde{\alpha} \leq \alpha$, $0<T<T_{\max }$, and assume further that $\Delta u_{0} \in C_{0}\left(\mathbb{R}^{N}\right), u_{0} \in C^{3}\left(\mathbb{R}^{N}\right)$ and

$$
\sup _{\substack{|\gamma| \leq 3 \\ x \in \mathbb{R}^{N}}}\left|\partial^{\gamma} u_{0}(x)\right|+\sup _{|\gamma|=3}\left|\partial^{\gamma} u_{0}\right|_{\widetilde{\alpha}}<\infty
$$

with the notation (1.7). It follows that $\Delta u \in C\left(\left[0, T_{\max }\right), C_{0}\left(\mathbb{R}^{N}\right)\right)$, that $\partial_{t} u, \nabla \partial_{t} u$, and all space-derivatives of $u$ of order $\leq 3$ belong to $C\left([0, T] \times \mathbb{R}^{N}\right)$, and that

$$
\begin{gathered}
\sup _{\substack{2 \ell+|\gamma| \leq 3 \\
0 \leq t \leq T}}\left\|\partial_{t}^{\ell} \partial_{x}^{\gamma} u(t)\right\|_{L^{\infty}}+\sup _{\substack{x \in \mathbb{R}^{N} \\
0 \leq t<s \leq T \\
2 \leq 2 \ell+|\gamma| \leq 3}} \frac{\left|\partial_{t}^{\ell} \partial_{x}^{\gamma} u(t, x)-\partial_{t}^{\ell} \partial_{x}^{\beta} u(s, x)\right|}{|t-s|^{\frac{\tilde{\alpha}+3-2 \ell-|\gamma|}{2}}} \\
+\sup _{\substack{0 \leq t \leq T \\
2 \ell+|\gamma|=3}}\left|\partial_{t}^{\ell} \partial_{x}^{\gamma} u(t)\right| \widetilde{\alpha}<\infty .
\end{gathered}
$$

In particular

$$
\sup _{\substack{0 \leq t \leq T \\|\gamma|=3}}\left|\partial_{x}^{\gamma} u(t)\right|_{\widetilde{\alpha}}<\infty
$$

In the above estimates, $\partial_{x}^{\gamma}=\partial_{x_{1}}^{\gamma_{1}} \cdots \partial_{x_{N}}^{\gamma_{N}}$ if $\gamma=\left(\gamma_{1}, \cdots, \gamma_{N}\right)$.

Proof. We define the Laplacian $A$ on $C_{0}\left(\mathbb{R}^{N}\right)$ by

$$
\left\{\begin{array}{l}
D(A)=\left\{u \in C_{0}\left(\mathbb{R}^{N}\right) ; \Delta u \in C_{0}\left(\mathbb{R}^{N}\right)\right\} \\
A u=\Delta u \quad u \in D(A) .
\end{array}\right.
$$

We equip $D(A)$ with the graph norm $\|u\|_{D(A)}=\|u\|_{L^{\infty}}+\|\Delta u\|_{L^{\infty}}$. It follows that $A$ is $m$-dissipative with dense domain and that $D(A) \hookrightarrow C_{0}^{1}\left(\mathbb{R}^{N}\right)$. More precisely

$$
\|\nabla u\|_{L^{\infty}} \leq C\|\Delta u\|_{L^{\infty}}^{\frac{1}{2}}\|u\|_{L^{\infty}}^{\frac{1}{2}}
$$

for all $u \in D(A)$. Indeed, consider the Bessel potential $G_{\sigma}, \sigma>0$. (See Aronszajn and Smith [3].) If $f \in C_{0}\left(\mathbb{R}^{N}\right)$, then $u=G_{2} \star f$ satisfies $-\Delta u+u=f, u \in C_{0}\left(\mathbb{R}^{N}\right)$ and $\Delta u \in C_{0}\left(\mathbb{R}^{N}\right)$. Moreover, since $\left\|G_{\sigma}\right\|_{L^{1}}=1$ (see [3, formula (4.6'), p. 417]) we see that if $u \in D(A)$, then $\|u\|_{L^{\infty}} \leq\|-\Delta u+u\|_{L^{\infty}}$. By an obvious scaling argument, we see that $\rho\|u\|_{L^{\infty}} \leq\|-\Delta u+\rho u\|_{L^{\infty}}$ for all $\rho>0$. Thus $A$ is $m$ dissipative. Furthermore, $C_{\mathrm{c}}^{\infty}\left(\mathbb{R}^{N}\right) \subset D(A)$ so that $D(A)$ is dense. To prove (A.5), consider $u \in D(A)$. We have $u=G_{2} \star(-\Delta u+u)$. Since $\nabla G_{2} \in L^{1}\left(\mathbb{R}^{N}\right)$ (see $[3$, formula (4.5), p. 417]), we deduce that $\|\nabla u\|_{L^{\infty}} \leq C\left(\|\Delta u\|_{L^{\infty}}+\|u\|_{L^{\infty}}\right.$ ), from which (A.5) follows by scaling.

Let now $u_{0} \in C_{0}\left(\mathbb{R}^{N}\right)$ and let $u \in C\left(\left[0, T_{\max }\right), C_{0}\left(\mathbb{R}^{N}\right)\right)$ be the corresponding maximal solution of (1.1). It follows from the above observations and from Pazy [16, Theorem 1.6, p. 187] that $u \in C^{1}\left([0, T], C_{0}\left(\mathbb{R}^{N}\right)\right) \cap C([0, T], D(A))$. In particular, using (A.5),

$$
\sup _{0 \leq t \leq T}\left(\|u(t)\|_{L^{\infty}}+\|\nabla u(t)\|_{L^{\infty}}\right)+\sup _{0 \leq t<s \leq T} \frac{\|u(t)-u(s)\|_{L^{\infty}}}{|t-s|}<\infty .
$$


Setting

and using the formula

$$
f=\lambda|u|^{\alpha} u
$$

$$
\nabla f=\lambda \frac{\alpha+2}{2}|u|^{\alpha} \nabla u+\lambda \frac{\alpha}{2}|u|^{\alpha-2} u^{2} \nabla \bar{u}
$$

we deduce from (A.6) that

$$
\begin{aligned}
\sup _{0 \leq t \leq T} & \left(\|f(t)\|_{L^{\infty}}+\|\nabla f(t)\|_{L^{\infty}}\right)+\sup _{0 \leq t<s \leq T} \frac{\|f(t)-f(s)\|_{L^{\infty}}}{|t-s|} \\
& +\sup _{0 \leq t \leq T}|f(t)|_{1}<\infty .
\end{aligned}
$$

In particular,

$$
\sup _{0 \leq t \leq T}\|f(t)\|_{L^{\infty}}+\sup _{0 \leq t<s \leq T} \frac{\|f(t)-f(s)\|_{L^{\infty}}}{|t-s|^{\frac{\tilde{\alpha}}{2}}}+\sup _{0 \leq t \leq T}|f(t)|_{\widetilde{\alpha}}<\infty .
$$

We apply Ladyzhenskaya et al. [14], Chapter IV, Section 2, p. 273, estimate (2.1) to the equations satisfied by the real and the imaginary parts of $u$, with $l=\widetilde{\alpha}$ and estimate (2.2) with $l=\widetilde{\alpha}+2$. It follows (among other properties) that

$$
\sup _{0 \leq t<s \leq T} \frac{\|\nabla u(t)-\nabla u(s)\|_{L^{\infty}}}{|t-s|^{\frac{\tilde{\alpha}+1}{2}}}+\sup _{0 \leq t \leq T}|\nabla u(t)|_{1}<\infty .
$$

We next recall the elementary estimate

$$
\begin{aligned}
\left.|| z_{1}\right|^{\alpha}-\left|z_{2}\right|^{\alpha} \mid & +\left.|| z_{1}\right|^{\alpha-2} z_{1}^{2}-\left|z_{2}\right|^{\alpha-2} z_{2}^{2} \mid \\
& \leq \begin{cases}C\left|z_{1}-z_{2}\right|^{\alpha} & 0<\alpha \leq 1 \\
C\left(\left|z_{1}\right|^{\alpha-1}+\left|z_{2}\right|^{\alpha-1}\right)\left|z_{1}-z_{2}\right| & \alpha \geq 1\end{cases}
\end{aligned}
$$

(See e.g. [7], formulas (2.26) and (2.27).) Estimate (A.9), formula (A.8), estimates (A.12), (A.6) and (A.11) imply that

$$
\begin{aligned}
\sup _{0 \leq t \leq T}( & \left.\|f(t)\|_{L^{\infty}}+\|\nabla f(t)\|_{L^{\infty}}\right)+\sup _{0 \leq t<s \leq T} \frac{\|f(t)-f(s)\|_{L^{\infty}}}{|t-s|^{\frac{1+\tilde{\alpha}}{2}}} \\
& +\sup _{0 \leq t<s \leq T} \frac{\|\nabla f(t)-\nabla f(s)\|_{L^{\infty}}}{|t-s|^{\frac{\tilde{\alpha}}{2}}}+\sup _{0 \leq t \leq T}|\nabla f(t)|_{\widetilde{\alpha}}<\infty .
\end{aligned}
$$

This allows us to apply again Ladyzhenskaya et al. [14], Chapter IV, Section 2, p. 273 , but this time we let $l=\widetilde{\alpha}+1$ in (2.1) and $l=\widetilde{\alpha}+3$ in (2.2). The conclusion follows.

\section{REFERENCES}

[1] Adams R.A. and Fournier J. J. F. Sobolev spaces. Second edition. Pure and Applied Mathematics (Amsterdam) 140. Elsevier/Academic Press, Amsterdam, 2003. (MR2424078)

[2] Alazard T. and Carles R. Loss of regularity for supercritical nonlinear Schrödinger equations, Math. Ann. 343 (2009), no. 2, 397-420. (MR2461259) (doi: 10.1007/s00208-008-0276-6)

[3] Aronszajn N. and Smith K. T. Theory of Bessel potentials. I. Ann. Inst. Fourier (Grenoble) 11 1961 385-475. (MR0143935) (link: http://www.numdam.org/item?id=AIF_1961__11__385_0)

[4] Bergh J. and Löfström J. Interpolation spaces. An introduction. Grundlehren der Mathematischen Wissenschaften 223. Springer-Verlag, Berlin-New York, 1976. (MR0482275) (doi: 10.1007/978-3-642-66451-9)

[5] Burq N., Gérard P. and Tzvetkov N. Multilinear eigenfunction estimates and global existence for the three dimensional nonlinear Schrödinger equations, Ann. Sci. École Norm. Sup. (4) 38 (2005), no. 2, 255-301. (MR2144988) (doi: 10.1016/j.ansens.2004.11.003) 
[6] Carles R. Geometric optics and instability for semi-classical Schrödinger equations, Arch. Ration. Mech. Anal. 183 (2007), no. 3, 525-553. (MR2278414) (doi: 10.1007/s00205-006-0017-5)

[7] Cazenave T., Fang D. and Han Z. Continuous dependence for NLS in fractional order spaces, Ann. Inst. H. Poincaré Anal. Non Linéaire 28 (2011), no. 1, 135-147. (MR2765515) (doi: 10.1016/j.anihpc.2010.11.005)

[8] Cazenave T. and Weissler F. B. The Cauchy problem for the critical nonlinear Schrödinger equation in $H^{s}$, Nonlinear Anal. 14 (1990), no. 10, 807-836. (MR1055532) (doi: $10.1016 / 0362-546 \mathrm{X}(90) 90023-\mathrm{A}$ )

[9] Christ M., Colliander J. and Tao T. Ill-posedness for nonlinear Schrödinger and wave equations, arXiv:math/0311048v1 [math.AP] (2003). (link: http://arxiv.org/abs/math/0311048)

[10] Fang D. and Han Z. On the well-posedness for NLS in $H^{s}$. J. Funct. Anal. 264 (2013), no. 6, 1438-1455. (MR3017270) (doi: 10.1016/j.jfa.2013.01.005)

[11] Kato T. On nonlinear Schrödinger equations, Ann. Inst. H. Poincaré Phys. Théor. 46 (1987), no. 1, 113-129. (MR0877998) (link: http://www.numdam.org/item?id=AIHPA_1987_-46_1_113_0)

[12] Kato T. Nonlinear Schrödinger equations, in Schrödinger Operators (Sønderborg, 1988), Lecture Notes in Phys. 345, Springer, Berlin, 1989, 218-263. (MR1037322) (doi: 10.1007/3-540-51783-9_22)

[13] Kato T. On nonlinear Schrödinger equations. II. $H^{s}$-solutions and unconditional wellposedness. J. Anal. Math. 67 (1995), 281-306. (MR1383498) (doi: 10.1007/BF02787794)

[14] Ladyzhenskaya O.A., Solonnikov V.A. and Ural'ceva N.N. Linear and quasilinear equations of parabolic type. Translated from the Russian by S. Smith. Translations of Mathematical Monographs, Vol. 23. American Mathematical Society, Providence, R.I. 1968. (MR0241821)

[15] Molinet L., Ribaud F. and Youssfi A. Illposedness issues for a class of parabolic equations, Proc. Royal Soc. Edinburgh Sect. A 132 (2002), 1407-1416. (MR1950814)

[16] Pazy A. Semi-groups of linear operators and applications to partial differential equations, Applied Math. Sciences 44, Springer, New-York, 1983. (MR0710486)

[17] Pecher H. Solutions of semilinear Schrödinger equations in $H^{s}$, Ann. Inst. H. Poincaré Phys. Théor. 67 (1997), no. 3, 259-296. (MR1472820) (link: http://www.numdam.org/item?id=AIHPA_1997_-67_3_259_0)

[18] Ribaud F. Cauchy problem for semilinear parabolic equations with initial data in $H_{p}^{s}\left(\mathbb{R}^{N}\right)$ spaces, Rev. Mat. Iberoamericana 14 (1998), no. 1, 1-46. (MR1639271) (doi: 10.4171/RMI/232)

[19] Tao T. Nonlinear dispersive equations. Local and global analysis. CBMS Regional Conference Series in Mathematics 106. Published for the Conference Board of the Mathematical Sciences, Washington, DC; by the American Mathematical Society, Providence, RI, 2006. (MR2233925)

${ }^{1}$ Université Pierre et Marie Curie \& CNRS, Laboratoire Jacques-Louis Lions, B.C. 187, 4 Place Jussieu, 75252 Paris Cedex 05, France

E-mail address: thierry.cazenave@upmc.fr

${ }^{2}$ Instituto de Matemática, Universidade Federal do Rio de Janeiro, Caixa Postal 68530, 21944-970 Rio de Janeiro, R.J., BRAZIL

E-mail address: flavio@labma.ufrj.br

${ }^{3}$ Université Paris 13, Sorbonne Paris Cité, CNRS UmR 7539 LAGA, 99 Avenue J.-B. Clément, F-93430 Villetaneuse, France

E-mail address: weissler@math.univ-paris13.fr 